Claremont Colleges

Scholarship@ Claremont

All HMC Faculty Publications and Research

HMC Faculty Scholarship

$1-1-2012$

\title{
Squaring, Cubing, and Cube Rooting
}

Arthur T. Benjamin

Harvey Mudd College

\section{Recommended Citation}

Benjamin, AT. Squaring, cubing and cube rooting. Coll Math J. 2012;43(1).

This Article is brought to you for free and open access by the HMC Faculty Scholarship at Scholarship @ Claremont. It has been accepted for inclusion in All HMC Faculty Publications and Research by an authorized administrator of Scholarship @ Claremont. For more information, please contact scholarship@cuc.claremont.edu. 


\title{
Squaring, Cubing, and Cube Rooting
}

\author{
Arthur T. Benjamin \\ Harvey Mudd College \\ Claremont, CA 91711 \\ benjamin@math.hmc.edu
}

I still recall my thrill and disappointment when I read Mathematical Carnival [4], by Martin Gardner. I was thrilled because, as my high school teacher had recommended, mathematics was presented in a playful way that I had never seen before. I was disappointed because it contained a formula that I thought I had "invented" a few years earlier. I have always had a passion for mental calculation, and the following formula appears in Gardner's chapter on "Lightning Calculators." It was used by the mathematician A. C. Aitken to mentally square large numbers.

\section{Squaring}

Aitken took advantage of the following algebraic identity.

$$
A^{2}=(A-d)(A+d)+d^{2}
$$

Naturally, this formula works for any value of $d$, but we should choose $d$ to be the distance to a number close to $A$ that is easy to multiply.

Examples. To square the number 23, we let $d=3$ to get

$$
23^{2}=20 \times 26+3^{2}=520+9=529 .
$$


To square 48 , let $d=2$ to get

$$
48^{2}=50 \times 46+2^{2}=2300+4=2304 .
$$

With just a little practice, it's possible to square any two-digit number in a matter of seconds. Once you have mastered those, we can quickly square three-digit numbers by rounding up and down to the nearest hundred.

Examples. $223^{2}=200 \times 246+23^{2}=49,200+529=49,729$

$$
952^{2}=1000 \times 904+48^{2}=904,000+2,304=906,304 .
$$

To do mental calculations of this size, one needs to be quick at multiplying 2-digit and 3-digit numbers by 1-digit numbers, generating the answer from left to right. More details and examples are given in $\mathrm{Se}$ crets of Mental Math [1], which Martin encouraged me to write. As I recently prepared a DVD version of this book [2], I learned some new methods for cubing and cube-rooting. Although much of that material did not end up in the DVD, I thought it would be of interest to readers of this Journal.

\section{Cubing}

To quickly cube a two-digit number in your head, it is worth learning how to multiply two two-digit numbers that are near each other. I call it the "close together method" and it is based on the algebra

$$
(z+a)(z+b)=z(z+a+b)+a b
$$


where $z$ is typically a number that ends in zero.

Example. For the problem $23 \times 26, z=20, a=3, b=6$, leads to

$$
23 \times 26=20 \times 29+3 \times 6=580+18=598 .
$$

Notice that on both sides of the equation, the algebra reveals that the two-digit numbers being multiplied will have the same sum $(23+$ $26=49=20+29)$.

Example. Thus, to do a problem like $88 \times 86$, since the numbers sum to $174=90+84$, we can begin with the product $90 \times 84$ :

$$
88 \times 86=90 \times 84+(-2) \times(-4)=7560+8=7568 .
$$

To cube a two-digit number, we can exploit the algebra

$$
A^{3}=(A-d) A(A+d)+d^{2} A \text {. }
$$

Example. Thus to cube the number 23, we can do

$$
23^{3}=20 \times 23 \times 26+3^{2} \times 23=20 \times 598+9 \times 23=11,960+207=12,167
$$

where we used the close-together method to do $23 \times 26$.

But there is a faster way to do this problem. Since we know that the problem will begin by doing $20 \times 29$, let's build that into the algebra. Writing the problem a different way, to cube the number $z+d$, we do

$$
(z+d)^{3}=z\left[z(z+3 d)+3 d^{2}\right]+d^{3} .
$$


Example. To cube $23, z=20$ and $d=3$ leads to the easier calculation

$23^{3}=20 \times[20 \times 29+27]+3^{3}=20 \times 607+3^{3}=12,140+27=12,167$.

This algebraic expansion is also known as Horner's method for polynomial evaluation. Notice that when cubing a two-digit number, $d$ will always be one of the numbers $\pm 1, \pm 2, \pm 3, \pm 4, \pm 5$ and so the number $3 d^{2}$ will always be one of the numbers $3,12,27,48$ or 75 , which simplifies the mental effort. Also notice that in the first two-digit multiplication, the number $z+3 d$ will always end with a digit that is three times the original last digit.

Example. For $88^{3}$, by tripling the last digit, we know that we will multiply our rounded number (90) by the number closest to 88 that ends in 4, namely 84. Thus,

$88^{3}=90 \times[90 \times 84+12]+(-2)^{3}=90 \times 7572-2^{3}=681,480-8=681,472$.

As a side note, the last part of the calculation is not as hard as it looks. By splitting $7572=7500+72$,

$$
9 \times 7572=(9 \times 7500)+(9 \times 72)=67,500+648=68,148 .
$$

To complete the calculation, simply multiply this number by 10 , and subtract 8 at the same time.

The calculation of two-digit cubes this way is surprisingly fast. With practice, the squares and cubes of two-digit numbers below 50 will be so quick that you will be able to cube three-digit numbers as well. 
Example. Exploiting the previous calculations:

$$
\begin{aligned}
323^{3} & =300 \times\left[300 \times 369+3\left(23^{2}\right)\right]+23^{3} \\
& =300 \times[110,700+1587]+23^{3} \\
& =3 \times 112,287 \times 100+23^{3} \\
& =33,686,100+12,167=33,698,267 .
\end{aligned}
$$

Another calculating tip. After the second multiplication, you can almost always say the millions part of the answer (here, 33 million) and hold the hundreds digit on your fingers. Here, just raise 1 finger to "hold onto" the hundreds digit so you only have to remember 686 while you calculate the cube of 23. By the way, if you just want a good cubing approximation, simply compute $z(z(z+3 d))$.

For example,

$$
323^{3} \approx 369 \times 300 \times 300=33,210,000 .
$$

This will come in handy in the calculation of cube roots.

\section{Cube Rooting}

One of the easiest feats of lightning calculation is determining the cube root of a perfect cube when the cube root is a two-digit number. The following description comes from Martin Gardner's classic book, Mathematics, Magic, and Mystery [5].

The cube root demonstration begins by asking members of the audience to select any number from 1 through 100, cube it, then call out the result. The performer 


\begin{tabular}{cccccccccc}
1 & 2 & 3 & 4 & 5 & 6 & 7 & 8 & 9 & 10 \\
\hline 1 & 8 & 27 & 64 & 125 & 216 & 343 & 512 & 729 & 1000 \\
& & \multicolumn{4}{c}{ TABLE 1. Table of Cubes }
\end{tabular}

instantly gives the cube root of each number called. To do the trick it is necessary first to memorize the cubes of the numbers from 1 through 10 .

An inspection of this table reveals that each cube ends in a different digit. The digit corresponds to the cube root in all cases except 2 and 3 and 7 and 8 . In these four cases the final digit of the cube is difference between the cube root and 10 .

To see how this information is used by the lightning calculator, let us suppose that a spectator calls out the cube root 250047 . The last number is a 7 which tells the performer immediately that the last number of the cube root must be 3 . The first number of the cube root is determined as follows. Discard the last three figures of the cube (regardless of the size of the number) and consider the remaining figures - in this example they are 250. In the above table, 250 lies between the cubes of 6 and 7 . The lower of the two figures - in this case 6 - will be the first figure of the cube root. The correct answer, therefore, is 63 .

One more example will make this clear. If the number called out is 19,683 , the last digit, 3 , indicates that the 
last digit of the cube root is 7 . Discarding the final three digits leaves 19, which falls between the cubes of 2 and 3. Two is the lower number, therefore we arrive at a final cube root of 27 .

I learned this trick in a book on mental magic [3] as a high school student, and wondered how it might be extended to larger problems, where the cube root was a three-digit number. I didn't pursue that question seriously at the time, since the cube of a three-digit number could be as large as nine digits, and most calculators back then only went up to eight digits. But now that calculators with greater capacity are quite common, I have learned two quick ways to do this problem, using casting out nines and casting out elevens.

\section{Casting Out Nines Method}

Ask someone to cube a three-digit number, and ask how many digits are in the answer. (It should be somewhere between seven and nine digits long.) Ask the volunteer to recite (or write down) the answer, and you can pretty easily determine the cube root in your head. The first digit and last digit of the cube root are determined exactly as before. The last digit of the cube root is uniquely determined by the last digit of the cube. (In fact, the last digit of the cube root is the last digit of the cube of the last digit of the cube!) For the first digit, we look at the magnitude of the millions.

Example. Let's find the cube root of the perfect cube 377,933,067. The first digit of the cube root must be 7 (since 377 lies between $7^{3}=$ 343 and $8^{3}=512$ ) and the last digit of the cube root must be 3 (since 


\begin{tabular}{c|ccccccccc}
$n$ & 0 & 1 & 2 & 3 & 4 & 5 & 6 & 7 & 8 \\
\hline$n^{3}$ & 0 & 1 & 8 & 0 & 1 & 8 & 0 & 1 & 8
\end{tabular}

TABle 2. Table of Cubes Mod 9

the cube ends in 7). Hence the answer must be of the form 7?3. How do we determine the middle digit? The method I use is to simply add the digits of the cube, mod 9 . Here the digits sum to 45 , which is a multiple of 9. This can only happen if the cube root is a multiple of three. In other words, the cube root must be 723 or 753 or 783 . Since 377 is much closer to 343 than 512, we would go with a cube root of 723 . For extra verification, we could estimate $720^{3} \approx 700 \times 700 \times 760=372,400,000$.

This same approach can be used when the cube is not a multiple of nine, since there are only three possible outcomes: if the cube root is 1 more than a multiple of 3 , then its cube is 1 more than a multiple of 9; if the cube root is 1 less than a multiple of 3 , then the cube is 1 less than a multiple of 9 , as seen in Table 2 .

Example. If the 3-digit cube results in 19,248,832, then we know that the cube root is of the form 2?8. To determine the middle digit, notice that the cube has digit sum 37, which reduces to $1 \bmod 9$. Hence the original number is congruent to $1 \bmod 3$, so the cube root must be either 208 or 238 or 268 or 298 . Judging from the proximity of 19 to its surrounding cubes, 8 and 27, the answer 268 seems most likely. Since $270^{3} \approx 300 \times 300 \times 210=18,900,000$, we have more confidence in our answer of 268.

\section{Casting Out Elevens Method}




\begin{tabular}{c|lllllllllll}
$n$ & 0 & 1 & 2 & 3 & 4 & 5 & 6 & 7 & 8 & 9 & 10 \\
\hline$n^{3}$ & 0 & 1 & 8 & 5 & 9 & 4 & 7 & 2 & 6 & 3 & 10
\end{tabular}

TABle 3. Table of Cubes Mod 11

With a little extra mental effort and memory, we can determine the middle digit without any guesswork, by realizing that the cubes of the numbers 0 through 10 are all distinct $\bmod 11$, as shown in Table 3 .

Example Revisited. For the cube root of $19,248,832$, we know that the answer will begin with 2 and end in 8 as in the last example. By alternately adding and subtracting its digits from right to left, we see that it reduces to $2-3+8-8+4-2+9-1=9(\bmod 11)$. Hence the cube root must reduce to $4(\bmod 11)$. Since the answer is of the form $2 ? 8$, then $8+2-?=4(\bmod 11)$ results in an answer of 268 , as well.

Example. To find the number with cube $111,980,168$, we see immediately that the answer will be of the form 4?2. Casting out 11s, we compute $8-6+1-0+8-9+1-1+1=3$. Here, the cube root must reduce to $9(\bmod 11)$. Thus $4+2-?=9(\bmod 11)$ tells us that the missing digit is 8 . Hence the cube root is 482 .

Between the two approaches, I prefer casting out nines over casting out elevens, since it is easier to add the digits of the cube as they are called out. I recommend that the reader play with a few examples using both methods, choose one method to master, and cast out the other one. 
Acknowledgment. The author wishes to thank the referee for many helpful suggestions, and especially Martin Gardner for being such an important factor in his life.

\section{REFERENCES}

[1] A. Benjamin and M. Shermer, Secrets of Mental Math: The Mathemagician's Guide to Lightning Calculation and Amazing Math Tricks, Random House, New York, 2006.

[2] A. T. Benjamin, The Secrets of Mental Math (DVD Course), The Teaching Company, Chantilly, VA, 2011.

[3] T. Corinda, 13 Steps to Mentalism, Tannen Magic Publishing, 4th Edition, 1968.

[4] M. Gardner, Mathematical Carnival: A New Round-up of Tantalizers and Puzzles from Scientific American, Random House, New York, 1965.

[5] M. Gardner, Mathematics, Magic, and Mystery, Dover Publications, New York, 1956. 